\title{
Saúde e meio ambiente: uma análise de diferenciais intra-urbanos enfocando o Município de São Paulo, Brasil*
}

\author{
Environment and health: an analysis of intra-urban differentials in the City of \\ S. Paulo, Brazil
}

Marco Akerman ${ }^{\star \star}$, Carolyn Stephens ${ }^{\star \star}$, Paulo Campanario ${ }^{\star \star \star}$, Paulo Borlina Maia ${ }^{\star \star \star}$

\begin{abstract}
AKERMAN, M. et al. Saúde e meio ambiente: uma análise de diferenciais intra-urbanos enfocando o Município de São Paulo, Brasil. Rev. Saúde Pública, 28: 320-5, 1994. Apresenta-se projeto na área de diferenciais intra-urbanos de saúde no Município de São Paulo, SP, Brasil. Focalizam-se alguns aspectos do problema urbano na cidade, seguindo-se uma revisão bibliográfica de trabalhos que abordam a questão dos diferenciais geográficos na literatura paulista. São outras temáticas: a desagregação geográfica do território a ser estudado e a metodologia de construção do índice de carência para definição de zonas homogêneas no Município de São Paulo, Brasil. Aponta-se qual seria a aplicação dos resultados finais obtidos pelo projeto.
\end{abstract}

Descritores: Saúde urbana. Mortalidade diferencial. Distribuição espacial.

\section{Introdução}

O ritmo acelerado do processo de urbanização em países em desenvolvimento tem levado a um crescente interesse no estudo de aspectos da saúde relacionados com o meio ambiente nas áreas urbanas desses países. Pesquisadores da "London School of Hygiene and Tropical Medicine (LSHTM)" Universidade de Londres, realizaram recentemente um levantamento, a pedido da Divisão de Desenvolvimento Urbano do Banco Mundial, sobre o impacto dos problemas do meio ambiente em relação a saúde das populações urbanas em países em desenvolvimento (Bradley e col. ${ }^{2}, 1992$ ).

A principal conclusão da citada pesquisa, confirmada pelos especialistas em saúde do Banco Mundial, foi que há lacunas no tipo de informação necessária sobre mortalidade para o estabelecimento de correlações entre saúde, urbanização e meio ambiente coletadas nas cidades dos países em desenvolvimento.

\footnotetext{
* Trabalho apresentado no IV Congresso Paulista de Saúde Pública, São Paulo, 1993.

** London School of Hygiene Tropical Medicine - Urban Health Unit - London, England

*** Fundação SEADE - Diretoria Adjunta de Estudos Populacionais, São Paulo, SP - Brasil
}

Separatas/Reprints: M. Akerman - CEDEC - Rua Airosa Galvão, 64 - 05002-070 - São Paulo, SP . Brasil

Edição subvencionada pela FAPESP. Processo 94/0500-0.
A partir do citado estudo, a "London School of Hygiene and Tropical Medicine", através da sua unidade de "Urban Health" apresentou projeto de pesquisa de campo a ser realizada em Acra e São Paulo, duas cidades que se contrastam em termos dos seus estágios de desenvolvimento, com o intuito de se compilar dados de mortalidade de doenças infecciosas, crônicas e psicossociais distribuídas por idade, sexo e áreas geográficas e correlacioná-los com variáveis socioeconômicas e do ambiente.

Mais especificamente, o projeto se propõe a: (1) documentar os diferenciais urbanos de mortalidade distribuídos por idade, sexo e área geográfica em duas cidades em estágios diferentes de desenvolvimento; e (2) documentar os diferenciais intraurbanos de algumas doenças infecciosas, crônicas e psicossociais em relação a categorias de carência ("deprivation") social e de ambiente.

O projeto foi aceito e está sendo financiado pelo Departamento de Meio Ambiente e Políticas da "Overseas Development Administration", órgão do Ministério das Relações Exteriores Britânico, com o apoio do Banco Mundial e suporte acadêmico da "London School of Hygiene and Tropical Medicine". A "etapa Acra" foi realizada em 1992 e a etapa "São Paulo" desenvolvida em colaboração com a Fundação SEADE, foi concluída em abril de 1994.

Focaliza-se no presente artigo alguns aspectos do problema urbano na cidade de São Paulo, seguindo-se uma revisão bibliográfica de trabalhos que abordam a questão dos diferenciais 
geográficos na literatura paulista. São abordados também os aspectos: referentes a desagregação geográfica do território a ser estudado e a construção do índice de carência para definição de zonas homogêneas no município.

\section{A cidade de São Paulo: alguns aspectos do problema urbano}

O crescimento de São Paulo tem gerado padrões urbanos similares aos de outras cidades da América Latina, onde grandes disparidades de fatores socioeconômicos e de saúde constituem uma das principais características desta megalópole (Jacobi ${ }^{13}, 1990$, Forattini $\left.{ }^{7}, 1991\right)$. Na verdade, a maioria dos trabalhadores ganha pouco, vive em precárias condições de moradia e perde horas num transporte público caro e ineficiente (Bogus ${ }^{1}$, 1991). Além disso, entre 1973 e 1987 houve um aumento de $1.031 \%$ na população das favelas em comparação com $61 \%$ de crescimento da população em geral (Bogus', 1991).

A distribuição da população no espaço urbano segue os padrões de desigualdade. De um lado, a periferia da cidade, sem infra-estrutura urbana básica, é ocupada por grupos menos privilegiados, de baixa renda e piores condições de saúde. Do outro, áreas com acesso total às facilidades urbanas, ocupadas por grupos de alta renda, vivendo em moradias satisfatórias e com melhores padrões de saúde (Jacobi $\left.{ }^{13}, 1990\right)$.

Apesar do conhecimento da magnitude da pobreza urbana, os impactos diferenciais dentro do espaço urbano provocados por diferentes condições socioeconômicas são pouco pesquisados (Stephens e col. $\left.{ }^{20}, 1991\right)$. Locker ${ }^{16}$ (1992) sugere que "medidas das características socioeconômicas por regiões são melhores indicadores de qualidade de saúde de subgrupos populacionais que medidas destas mesmas características em indivíduos ou famílias".

\section{Estudos sobre diferenciais geográficos em São Paulo}

Pesquisadores locais já desenvolveram importantes trabalhos para medir diferenciais entre áreas geográficas.

O trabalho pioneiro de Leser ${ }^{14}$ (1972) associa algumas características geográficas e históricas dos distritos e subdistritos do Município de São Paulo com a mortalidade infantil, subdividindoos em três anéis geográficos, central, intermediário e periférico, de acordo com essas características.
Estudo minucioso e abrangente publicado pela FUNDAP (Fundação de Desenvolvimento Administrativo ${ }^{8}, 1983$ ) mostrou diferenciais regionais para o Estado de São Paulo. Eles utilizaram 20 variáveis socioeconômicas e 9 variáveis relacionadas com estatísticas de mortalidade para derivar áreas homogêneas para estes dois atributos. Uma matriz dessas áreas homogêneas relacionou variáveis socioeconômicas e estatísticas de mortalidade e encontrou: (1) padrões semelhantes de mortalidade para diferentes condições socioeconômicas; (2) diferenças entre os componentes dos mesmos grupos homogêneos; (3) regiões de perfil socioeconômico mais favorável apresentando maiores taxas de mortalidade para doenças associadas com o desenvolvimento. Não há achados relativos a sexo ou grupo etário.

Ferreira e Grillo ${ }^{6}$ (1983), usando a técnica de análise de conglomerados, agregam áreas homogêneas do Estado de São Paulo de acordo com padrões de taxa de mortalidade infantil. Contudo, eles não relacionaram esses padrões com nenhuma condição socioeconômica.

Bussab e Dini ${ }^{3}$ (1985), em estudo muito citado na literatura de diferenciais geográficos, dividiram São Paulo (cidade e área metropolitana) em regiões homogêneas por perfil ocupacional. Não há associação com indicadores de saúde nesse estudo. Em 1988/9 esse mesmo estudo foi publicado em formato monográfico pela Fundação SEADE, que enfatiza a necessidade de estudos semelhantes para apoiar atividades de planejamento.

Sobral ${ }^{19}$ (1988) apresenta 10 mapas da cidade de São Paulo onde distribui, pelos distritos e subdistritos, a mortalidade proporcional por várias doenças infecciosas e crônicas, para dar subsídios a "uma geografia médica da cidade". Na descrição de cada mapa, foram feitas breves associações com características socioeconômicas dos distritos e subdistritos da cidade.

A Fundação SEADE ${ }^{11}$, em 1988, mostra as condições de vida de crianças e jovens $(0$ a 19 anos) na cidade e áreas metropolitanas. $\mathrm{Na}$ primeira parte foram apresentadas diferenciais intraurbanos relacionados com migração, renda, tamanho da família, saneamento, taxas de fertilidade, ocupação, participação feminina no mercado de trabalho e educação. Procurou-se agrupar áreas em grupos homogêneos e associar variáveis socioeconômicas com indicadores de saúde. $\mathrm{Na}$ segunda parte, foram agrupadas taxas de mortalidade infantil em quatro grupos homogêneos e apresentada tabela associando educação da mãe e mortalidade por causa específica. Entretanto, não houve associação explícita de diferenciais socioeconômicos baseados em área com diferenciais de mortalidade infantil baseados em área. Foram 
apresentadas também taxas de mortalidade desagregadas por idade $(1-4 ; 5-9 ; 10-14 ; 15-19)$, sexo e causa específica de morte. Diferente da análise da primeira parte (mortalidade infantil), não houve đesagregação por área geográfica (Fundação SEADE $\left.{ }^{11}, 1988\right)$.

O estudo de Ramos e Goihman ${ }^{17}$ (1989) apresentou uma estratificação geográfica por padrão socioeconômico em 48 subdistritos da cidade de São Paulo. Classificaram as áreas de acordo com renda média familiar, serviço de esgoto e proporção de idosos (65 ou mais) na população total. Não há relação desses diferenciais socioeconômicos com indicadores de saúde e o trabalho parece ter sido elaborado como proposta de amostragem para um estudo posterior da população idosa.

Seguindo a mesma linha da publicação sobre crianças e jovens ${ }^{11}$, a Fundação SEADE ${ }^{10}$ (1990) desenvolveu estudo descrevendo as condições de moradia da população idosa na área metropolitana de São Paulo. Foram apresentados padrões geográficos da proporção de idosos agrupados em áreas homogêneas, associando essas áreas homogêneas com vários indicadores demográficos e socioeconômicos (estado civil, educação, nível de atividade, perfil ocupacional e renda). Também foram descritas taxas de mortalidade por causa específica desagregadas por idade e sexo e proporção de algumas doenças afetando a população mais velha que vive na comunidade. Não há estratificação geográfica por taxas de mortalidade.

Destacam-se dois trabalhos apresentados no II Congresso Brasileiro de Epidemiologia (julho, 1992) que mostram duas análises utilizando estatísticas de mortalidade desagregadas e indicadores socioeconômicos. Lira e col. ${ }^{15}$ (1992) encontraram taxas de mortalidade por câncer de colo mais altas na periferia da cidade e nas mulheres com nível de instrução mais baixo. Gawryszewski e Drumond $\mathrm{Jr}^{12}$ (1992) demonstraram que a mortalidade por causas externas (acidentes de trânsito, homicídios e suicídios) é a causa mais significativa de morte no grupo de 5 a 39 anos. Demonstraram ainda que a taxa de mortalidade por homicídios é de $11,3 / 100.000$ no Jardim Paulista (área rica) e 69,2/100.000 no Itaim Paulista (área pobre).

Uma publicação da Secretaria de Estado de Planejamento ${ }^{18}$ (1993) aborda o tema de construir medidas compostas para as várias regiões do Estado de São Paulo. Agregam taxas de mortalidade infantil, taxas de mortalidade geral e proporção de mortes causadas por doenças infecciosas para derivar o índice "Condições de Saúde". Usam duas variáveis relacionadas com educação para criar o índice "Condições de Educação", e, combinando esses dois índices, encontram um índice geral de- nominado "Condições Sociais". As diferenças regionais são apresentadas com base nesses índices.

Apesar dos estudos locais abordarem vários aspectos importantes da área de diferenciais geográficos, há ainda algumas lacunas a serem preenchidas. Em primeiro lugar, os trabalhos até agora concentraram mais em diferenciais regionais do que diferenciais intra-urbanos. Provavelmente o espaço urbano é mais sensível para mostrar diferenças sociais do que o espaço regional. Além disso, não houve tentativa sistematizada para descrever diferenciais por sexo e todos grupos etários em relação ao grupo social. Por exemplo, não há estudo publicado comparando padrões de mortalidade com condições socioeconômicas para a população adulta (19 a 60), desagregados para todas as regiões geográficas dentro do espaço urbano. $\mathrm{E}$, finalmente, mesmo quando mortalidade e diferenciais socioeconômicos foram descritos, há importantes lacunas quanto a sexo, idade e desagregação geográfica.

\section{Desagregação geográfica do Município de São Paulo}

Estatísticas agregadas podem camuflar diferenças importantes. Por exemplo, a taxa de mortalidade infantil no Município de São Paulo, em 1992 , de 25,2 por mil nascidos vivos, estaria representando um retrato médio do problema na cidade. Taxas relativamente baixas, como por exemplo, de 12,6 por mil no subdistrito de Indianópolis, podem conviver com taxas até 3 vezes maiores, como a do Itaim Paulista, de 38,1*.

Este tipo de exercício desagregado da estatística clamaria por intervençōes diferenciadas entre os diversos territórios. E como administrar é conviver com limitação de recursos, estatísticas desagregadas como essas poderiam servir para que, através de uma discriminação positiva, o investimento pudesse ser priorizado na direção daquelas áreas onde o problema tivesse maior magnitude.

Sendo um dos objetivos do presente projeto obter resultados que direcionem agências locais e internacionais na identificação de grupos-alvo para a alocação de recursos, a questão da desagregação geográfica "ótima" se apresenta como tema relevante. Em outras palavras, qual seria a menor desagregação geográfica possível que pudesse obter áreas mais homogêneas do ponto de vista socioeconômico e de ambiente no Município de São Paulo?

\footnotetext{
* Dados fornecidos pela Fundação SEADE.
} 
Claro está que este princípio tem um limite, pois as sucessivas desagregações levariam nos finalmente a endereços de famílias, e nesse caso extremo e hipotético, seu estudo não conduziria a conclusões derivadas de áreas geográficas. Ademais, os dados não permitiriam generalizações significativas pela quantidade pequena de acontecimentos ocorridos num determinado período de tempo. Eames e col. ${ }^{5}$ (1992) contribuem com essa discussão dizendo que "a desagregação geográfica mínima deve representar um compromisso entre obter uma área reduzida o suficiente para ser homogênea e extensa o bastante para fornecer número adequado de mortes para análise".

Numa cidade como São Paulo, a situação ideal seria a de se ter pequenas áreas que diferenciassem claramente as condições de vida de grupos relativamente homogêneos do ponto de vista residencial (áreas de favelas, de cortiços, entre outras). Entretanto, a tradição de coleta de informações vitais e socioeconômicas na cidade não vêm considerando como bases geográficas áreas que reflitam grupos homogêneos da população, mas territórios de jurisdição de cartórios de registro civil ou de administração pública. Por exemplo, o subdistrito de Butantã, área de jurisdição de um cartório de registro civil, abriga simultaneamente domicílios habitados por famílias de alta renda e favelas onde moram famílias de baixa renda. O Município de São Paulo possui 56 distritos e subdistritos que são as tradicionais bases geográficas onde se compatibilizam dados censitários e do movimento do registro civil.

A lei $n^{\circ} 11.220$ de 20 de maio de 1992 formalizou uma nova divisão, que divide o espaço municipal em 96 distritos. Estas novas unidades foram criadas com o intuito de se aproximar de uma maior homogeneidade geográfica, além de se criar áreas comuns de administração pública. Subdistritos como o Butantã, anteriormente constituído enquanto uma área única, passa nesta nova divisão a se dividir em 6 novos territórios o que provavelmente produz espaços com maior grau de homogeneidade.

No entanto, as vantagens dessa nova divisão têm que ser ponderadas com suas desvantagens, muitas das quais são de caráter temporário. O primeiro problema é o da compatibilidade com a antiga segmentação (56 distritos e subdistritos). Com efeito, nem sempre os distritos gerados pela nova divisão são subconjuntos perfeitos da divisão territorial anterior. Este fato traz consequiências para o acompanhamento desagregado da série histórica da mortalidade no município porque dificulta a distribuição, na nova repartição, dos eventos anteriormente ocorridos atribuídos à antiga divisão. Apesar das unidades censuais mínimas serem compatíveis com a nova partilha do município, não há ainda disponibilidade de novos eventos vitais distribuídos pela nova divisão.

A vista dos problemas acima assinalados, utilizou-se como unidade básica de análise a divisão territorial de 56 distritos e subdistritos. No entanto, na medida do possível, os poucos dados disponíveis atualmente para a nova segmentação serão trabalhados para detectar possíveis heterogeneidades na antiga divisão.

\section{Escolha das variáveis socioeconômicas e de ambiente, que comporão o índice de carência para a definição das zonas homogêneas no Município de São Paulo}

Os índices compostos de carência são instrumentos que possibilitam demonstrar o fenômeno em áreas geográficas específicas, classificandoas em termos de variáveis que reflitam as circunstâncias materiais ou sociais da área em questão (Carstairs and Morris ${ }^{4}$, 1989; Locker $^{16}$, 1992). Ao se combinar vários indicadores como referenciais para a descrição de áreas geográficas, presume-se a interação de vários fatores na determinação da qualidade de vida daquela área. A questão é identificar porque certos efeitos ocorrem mais em certas populações do que em outras e o objetivo está em apontar os determinantes destes efeitos em relação às características de grupos populacionais e não de indivíduos.

Afim de se decidir quais variáveis deveriam ser incluídas no índice de carência a ser usado no Município de São Paulo, organizou-se um seminário com a participação de planejadores de 12 instituições públicas municipais e estaduais.

Foram formados dois grupos de trabalho e utilizando-se o método "técnica de Delfos"* procedeuse a escolha de 5 variáveis. Estas foram escolhidas a partir de uma lista de variáveis obtidas dos documentos e estudos disponíveis em São Paulo, as quais têm sido associadas em estudos internacionais e locais como indicadores de carência social e de ambiente.

Esta lista, que não se pretendeu fosse completa, representava um amplo espectro de possibilidades tendo como critério a disponibilidade da informação. Cada participante, baseando-se nas dis-

\footnotetext{
* O nome desta técnica inspira-se no Oráculo de Delfos usado pelos gregos antigos para predizer o futuro. Esta técnica vem sendo aplicada para desenvolver prognósticos de eventos futuros baseados na opiniāo coletiva de especialistas. Estas opiniōes são então compiladas e reapresentadas para os participantes; caso eles sintam necessidade, podem revisar suas opiniões anteriores. Esta decisão final forma então a opinião do grupo.
} 
cussões de grupo, ordenou essas variáveis de acordo com sua própria opinião sobre quais seriam as melhores preditoras de carência social.

Dois critérios foram sugeridos para a escolha das variáveis. $O$ primeiro, a validade da variável, isto é, a sua relevância ou importância como medida de carência em São Paulo; e o segundo, a confiabilidade da variável, que poderia ser definida como uma medida de qualidade do dado, ou em outras palavras, "se podemos confiar na informação gerada pelo indicador".

Uma vez completado este exercício, todos os formulários foram computados, e foi produzida uma classificação geral das variáveis. Discutiu-se em plenária esta classificação, e foi facultado aos participantes a revisão da lista preliminar.

Terminado este exercício, foram apresentadas as 5 variáveis que irão compor o índice de carência a ser usado pelo projeto. A tabela apresenta a classificação final de todas as variáveis.

Tabela - Classificação final de todas as variáveis.

\begin{tabular}{|c|c|c|c|}
\hline \multirow{4}{*}{$\begin{array}{l}N^{2} \\
1 \\
2 \\
3\end{array}$} & \multirow{4}{*}{\begin{tabular}{l}
\multicolumn{1}{c}{ Indicadores } \\
Renda familiar per capita \\
Acessibilidade ao emprego \\
Proporção de população não econo- \\
micamente ativa
\end{tabular}} & \multicolumn{2}{|c|}{ Classificação } \\
\hline & & $\rightarrow$ & 1 \\
\hline & & & 8 \\
\hline & & & 11 \\
\hline 4 & Abastecimento de água & & 6 \\
\hline 5 & Consumo de água per capita & $\rightarrow$ & 4 \\
\hline 6 & Rede de esgoto & $\rightarrow$ & 3 \\
\hline 7 & $\begin{array}{l}\text { Percentagem de população analfabeto } \\
\text { e com primário incompleto }\end{array}$ & $\rightarrow$ & 2 \\
\hline 8 & Padrāo da área construida & & 9 \\
\hline 9 & Erosāo e inudação & & 12 \\
\hline 10 & Sexo do chefe da familia & & 10 \\
\hline 11 & Número de pessoas por domicilio & $\rightarrow$ & 5 \\
\hline 12 & $\begin{array}{l}\text { Metros quadrados de construção per } \\
\text { capita }\end{array}$ & & 7 \\
\hline 13 & Proporção de migrantes & & 13 \\
\hline
\end{tabular}

\section{Uso dos resultados}

O uso de informação em saúde já existente poderia fornecer a base de um quadro amplo dos diferenciais de saúde intra-urbanos o que, com certa cautela, daria a possibilidade de descrevê-los em relação a variáveis socioeconômicas e do ambiente. A vantagem de se utilizar dados já existentes é que se pode realizar a tarefa mais rapidamente e não se requer extensiva e onerosa coleta primária de dados. Mais ainda, esta metodologia que emprega dados já disponíveis em cada cidade para se descrever o ônus intra-urbano em relação à saúde, faria com que outros governos munic'? iais em países em desenvolvimento pudessem reproduzir a experiência de forma econômica. Este método poderia ainda ser utilizado na identificação de grupos de risco em relação às condições do ambiente, avaliando diferenciais intra-urbanos e priorizando alocação de recursos.

Pode-se antecipar que os resultados finais do projeto poderão ter a seguinte aplicação:

- identificação de opções de gerenciamento urbano e do ambiente através do esclarecimento das relações entre condições socioeconômicas, meio ambiente e saúde.

- acréscimo ao conhecimento das relações entre saúde e meio ambiente a compreensão da dimensão e das características dos diferenciais intra-urbanos de saúde em duas cidades de países em desenvolvimento.

- direcionamento de agências locais e internacionais, na identificação de grupos-alvo para a alocação de recursos relativos ao meio ambiente.

- demonstração da reprodutibilidade da metodologia que emprega informação já existente em saúde e meio ambiente na identificação de grupos de risco e dos fatores socioeconômicos e de ambiente associados a esses grupos.

- geração de hipóteses sobre condições urbanas específicas de saúde e suas associações com o meio ambiente, que sejam significativas no nivel local.

- orientação quanto à elaboração de políticas no sentido da relevância da ação multisetorial ao se abordar a questão da qualidade de vida da população urbana pobre.

\section{Agradecimentos}

Agradecemos à Faculdade de Saúde Pública da USP, ao Instituto de Saúde da Secretaria de Estado da Saúde, à Fundação para o Desenvolvimento da Educação, à SABESP, à CETESB, à Secretaria Municipal de Saúde, à Secretaria Municipal de Planejamento, à Secretaria Municipal de Habitação e Desenvolvimento Urbano, ao Centro de Estudos de Cultura Contemporânea (CEDEC), ao Instituto de Pesquisas Tecnológicas da USP e ao Centro Nacional de Epidemiologia do Ministério da Saúde pela participação de seus profissionais no Seminário Introdutório do Projeto, onde foram escolhidas as variáveis socioeconômicas e de ambiente que serão usadas na definição das zonas homogêneas no Município de São Paulo.

AKERMAN, M. et al. [Environment and health: an analysis of intra-urban differentials in the City of S. Paulo, Brazil]. Rev. Saúde Pública, 28: 320-5, 1994. A project concerned with the study of intra-urban health differentials in S. Paulo city, Brazil, is described. A brief outline of urban problems in the city is presented, followed by a review of the locally published literature on the subject 
of geographical differentials (stratification by socioeconomic status and environment). Two topics are introduced: geographical subdvision of the area studied and the methods used to choose the socio-environmental indicators for the construction of a deprivation index with to stratify the city. Suggestions are made as to possible applications of results achieved by the project.

Keywords: Urban health. Differential mortality. Residence characteristics.

\section{Referências Bibliográficas}

1. BOGUS, L.M.M. Direito à cidade e segregação espacial. $S$. Paulo Perspect. 5: 47-50, 1991.

2. BRADLEY, D.; STEPHENS, C.; HARPHAM, T.; CAIRNCROSS, S. A Review of environmental health impacts in developing countries cities. Washington, World Bank, 1992.

3. BUSSAB, W.O. \& DINI, N.P. Pesquisa de emprego e desemprego SEADE/DIEESE: regiōes homogêneas da Grande São Paulo. S. Paulo Perspect., 1: 5-11, 1985.

4. CARSTAIRS, V. \& MORRIS, R. Deprivation and mortality: an alternative to social class? Community Med., 11: 210-9, 1989.

5. EAMES, M.; BEM SCHLOMO, Y.; MARMOT, M. Health education project: area deprivation and premature mortality. Londres, Department of Epidemiology and Public Health, University of London, 1992.

6. FERREIRA, C.E.C. \& GRILLO, J.E. Níveis e padróes da mortalidade infantil nas sub-regiões do Estado de São Paulo. Inf. Demogr., 8: 137-54, 1983.

7. FORATTINI, O.P. Qualidade de vida e meio urbano: a cidade de São Paulo, Brasil. Rev. Saúde Pública, 25: 75. $86,1991$.

8. FUNDAÇÃO DE DESENVOLVIMENTO ADMINISTRATIVO. Características regionais de saúde no Estado de São Paulo, São Paulo, FUNDAP, 1983.

9. FUNDAÇÃO SEADE. Regiões homogêneas: um estudo dos perfis ocupacionais do interior. São Paulo, 1988

10. FUNDAÇÃO SEADE. O idoso na Grande São Paulo. São Paulo, 1990.
11. FUNDAÇÃo SEADE. O jovem na Grande São Paulo. São Paulo, 1988.

12. GAWRYSZEWSKI, V.P. \& DRUMOND Jr., M. A morte violenta no município de São Paulo em 1991. In: Relatório de participação da Secretaria Municipal de Saúde no II Congresso Brasileiro de Epidemiologia. São Paulo, Secretaria Municipal de Saúde, 1992.

13. JACOBI, P. Habitat e saúde na periferia. S. Paulo Perspec., 4: $121-30,1990$.

14. LESER, W.S.P. Relacionamento de certas características populacionais com a mortalidade infantil no município de Sāo Paulo. Probl. Bras. 10(109): 17-33, 1972.

15. LIRA, M.M.T. de A.; DRUMOND Jr., M.; GUTIERREZ, E.B. A desigualdade no perfil do câncer ginecológico no município de São Paulo. In: Relatório de participação da Secretaria Municipal de Saúde no II Congresso Brasileiro de Epidemiologia. São Paulo, Secretaria Municipal da Saúde, 1992

16. LOCKER D. Measuring social inequality in dental health services research: individual, household and areabased measures. Toronto, Department of Community Dentistry and Community Dental Health Services Research Unit, University of Toronto, 1992.

17. RAMOS, L.R. \& GOIHMAN, S. Geographical stratification by socio-economic status: methodology from a household survey with elderly people in S. Paulo, Brazil, Rev. Saúde Pública, 23: 478-92, 1989.

18. SECRETARIA ESTADUAL DE PLANEJAMENTO E GESTÃO. Como estāo os paulistas? Indicadores de renda per capita e condiçōes sociais. In: Como está São Paulo? São Paulo, Secretaria Estadual de Planejamento e Gestão, 1993. p. 33-48.

19. SOBRAL, H.R. Mapeamento das causas de morte no município de São Paulo: subsídios a uma geografia médica da cidade. Bol. Paul. Geogr., 66: 85-96, 1988.

20. STEPHENS, C.; HARPHAM, T.; CAIRNCROSS, S.; TIMAEUS, I. Environment and health in developing countries: an analysis of intra-urban differentials. Londres, London School of Hygiene and Tropical Medicine, 1991.

Recebido para publicação em 26.8 .1993 Reapresentado em 25.5.1994 Aprovado para publicaşão em 7.6.1994 